\title{
A rare case of utero peritoneal fistula
}

\section{Yendru Katyayani Swapna*, Savitha Devi Yelamanchi, Kamalakar Naidu Talipineni, Polavarapu Jyothsna}

Department of Obstetrics and High-risk Pregnancy, Swapna Health Care, Hyderabad, Telangana, India

Received: 20 February 2018

Accepted: 27 March 2018

\section{*Correspondence:}

Dr. Yendru Katyayani Swapna,

E-mail: swapnahealthcare@gmail.com

Copyright: (C) the author(s), publisher and licensee Medip Academy. This is an open-access article distributed under the terms of the Creative Commons Attribution Non-Commercial License, which permits unrestricted non-commercial use, distribution, and reproduction in any medium, provided the original work is properly cited.

\section{ABSTRACT}

Uterine fistulas are very rare and can be due to gynaecological surgery, trauma or infections. 17 year old unmarried girl presented with uteroperitoneal fistula, following left salpingo-oophorectomy for torsion of left adnexae done one year ago outside. She came with increasing dysmenorrhea since surgery. USG \& MRI showed cystic mass left cornual area. Vaginoscopy and Hystero-laparoscopy showed a thick longitudinal vaginal septum, uni-cornuate uterus with left horn non-communicating with cervix but open into the peritoneal cavity, the false passage being the result of previous surgery. Excision of vaginal septum and laparoscopic excision of the horn and repair provided very good relief, till now (8months from surgery).

Keywords: Dysmenorrhoea, Laparoscopy, Uteroperitoneal fistula, Vaginal septum

\section{INTRODUCTION}

Uterine fistulas are rare pathologic entities that are characterised by abnormal communication of uterine cavity with any other organ or structure through a perforation formed due to gynaecologic surgery, trauma or infections. Exact incidence is not known. ${ }^{1}$ Lower segment caesarean section has increased the prevalence of these uterine fistulae and accounts for $83 \%$ of cases. ${ }^{2}$

Rarely, utero-peritoneal fistula could be related to prolonged labour, forceps delivery, vaginal birth after caesarean section, gynaecological injuries, tuberculosis of the genital tract and intrauterine contraceptive devices. ${ }^{3}$

Utero-peritoneal fistula has been reported following scar dehiscence after laparoscopic myomectomy. ${ }^{1}$

Use of electro surgery for haemostasis inside uterine defect may also increase risk of uteroperitoneal fistula formation1. Colouterine fisulae secondary to sigmoid diverticulitis have been reported. ${ }^{4}$

\section{CASE REPORT}

17-year-old unmarried girl presented to our outpatient department in May 2017 with complaints of severe dysmenorrhoea of increasing intensity since 1year, not responding to medical treatment. She underwent laparoscopic surgery 1year ago for chocolate cysts in left ovary, at a different hospital. The discharge summary mentioned broad fundus, torsion of left adnexae with multiple chocolate cysts and mass adherent to colon and uterus. Left salpingo oophorectomy was done and she received 3 doses of Inj. Lupride post operatively.

USG showed a cystic lesion with another small adjacent hyperechoic lesion in soft tissues measuring about $30 \times 24 \mathrm{~mm}$ in left adnexa, and uterus septate/ bicornuate with left rudimentary horn.

MRI was done and incomplete fusion of bilateral uterine horns with the left uterine horn not communicating with cervix was noted. Hyperintense lesions seen abutting uterine fundus and at level of isthmus of left uterine horn 
suggestive of endometriotic cyst. CA-125 was raised $(158 \mathrm{U} / \mathrm{ml})$. She was advised hysteron-laparoscopy and excision of non-communicating rudimentary horn, and detailed counselling was done, and consents taken. Intra Operative findings: She had a thick muscular longitudinal vaginal septum (Figure 1).

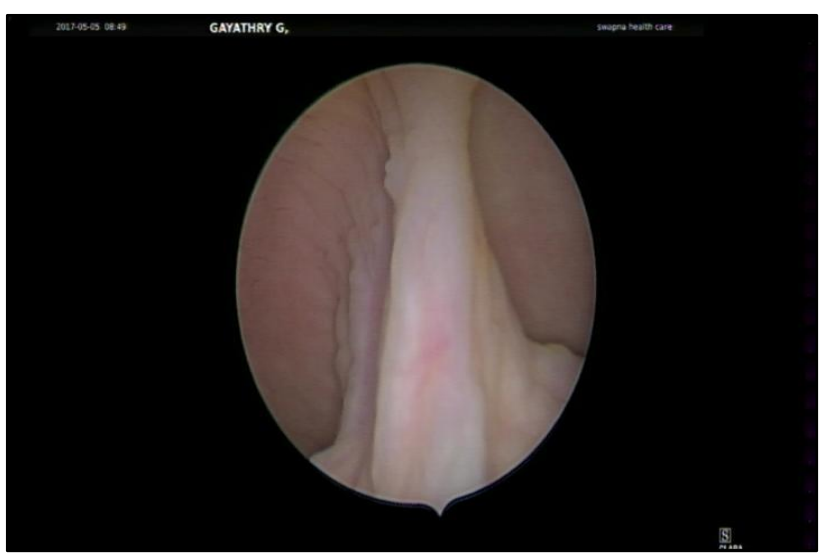

Figure 1: Longitudinal vaginal septum.

The vaginal septum was completely excised Figure 2.

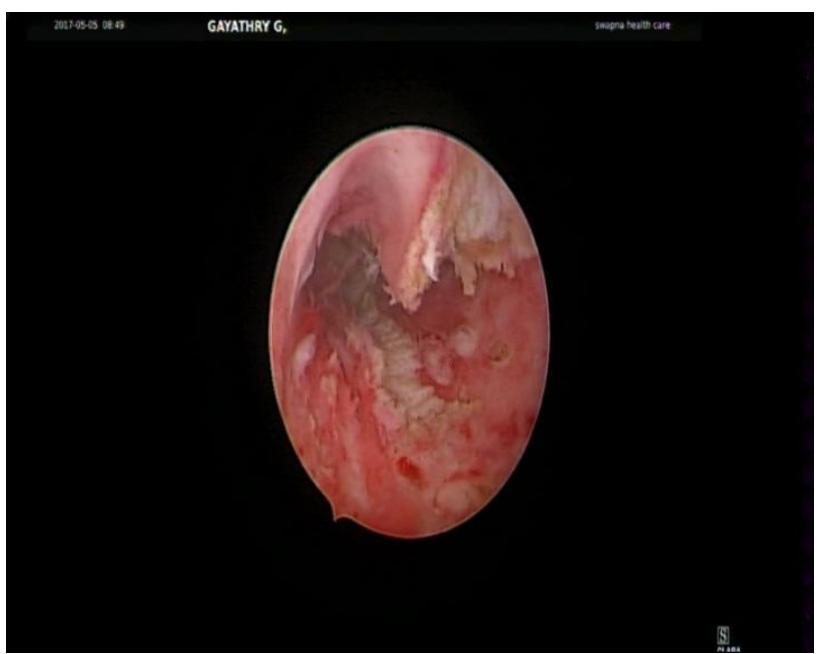

Figure 2: Vaginal septum resected.

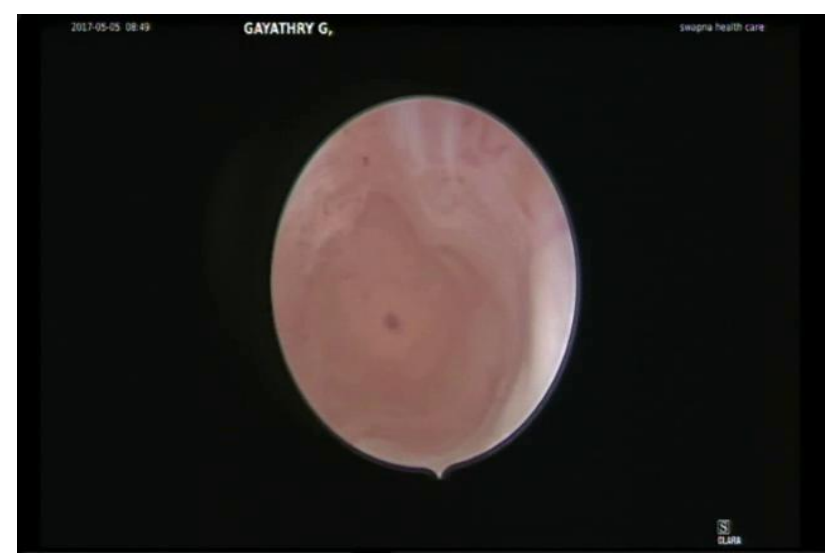

Figure 3: Single horn with ostium at apex.
Hysteroscopy revealed a unicornuate uterus with centrally placed ostium Figure 3 . There were omental and bowel adhesions to the left cornual area Figure 4. Both uterosacral ligaments were attached to the main right horn. Left Fallopian tube and ovary were absent. Right fallopian tube, ovary and right part of uterus were normal.

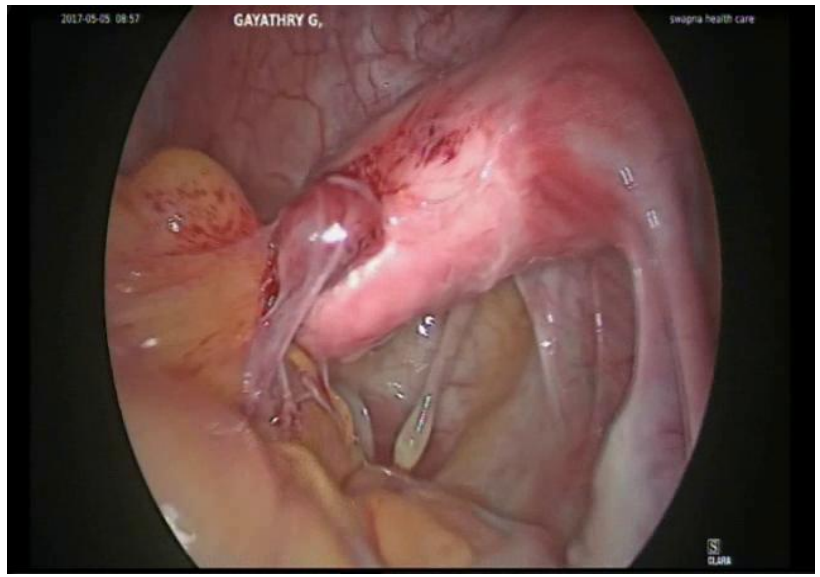

Figure 4: Absent left adnexae with adhesions to left horn.

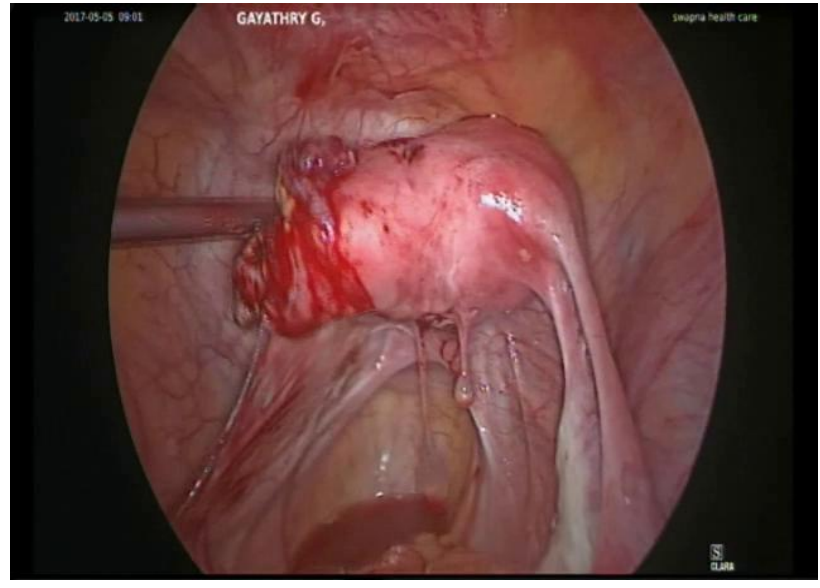

Figure 5: Uteroperitoneal fistula.

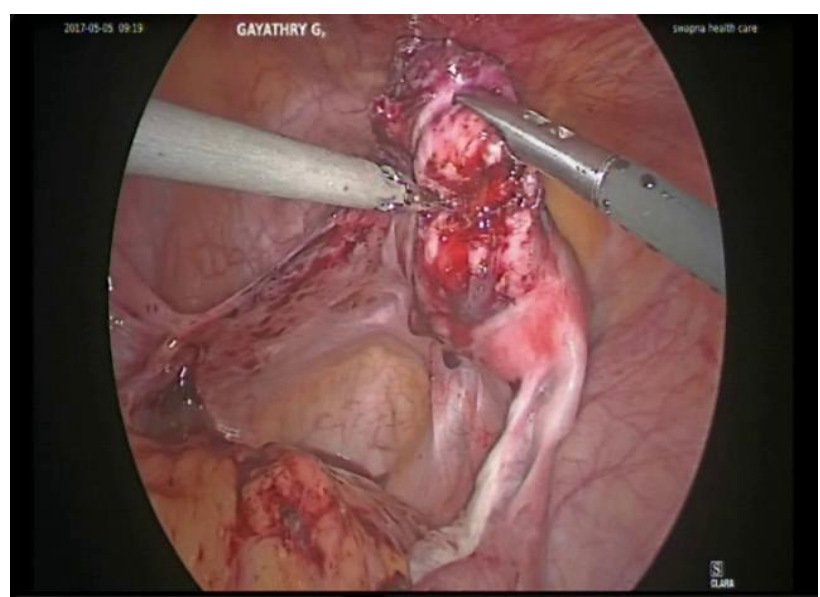

Figure 6: Excision of non-communicating horn. 


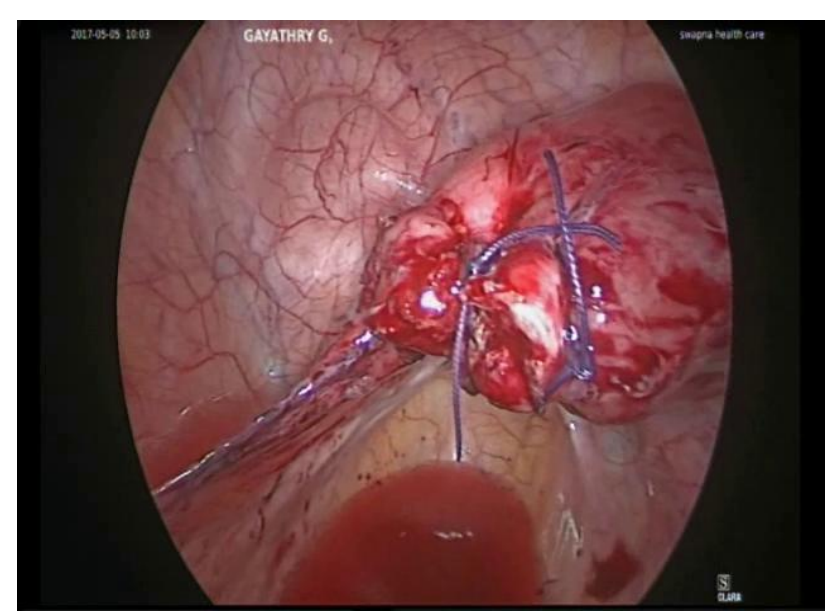

Figure 7: After repair and reconstruction.

On removal of adhesions, there was a noncommunicating left horn which was open at the cornual area, leaking dark chocolate coloured fluid into peritoneal cavity, indicating a uteroperitoneal fistula Figure 5.

Excision of Figure 6 and 7 left horn was done. Myometrium was sutured with vicryl, closing the defect. Follow-up for eight months showed no recurrence of mass and she had total relief from dysmenorrheoa.

\section{DISCUSSION}

Uteroperitoneal fistula is a rare entity. Exact occurrence is not known, with only a few reported cases. It can occur after LSCS, prolonged labour, forceps delivery, VBAC, Gynaecologic injuries, TB of genital tract and perforation with IUCD3. It can also occur after uterine artery embolization5, after sterilization operation if carried out near proximal end of fallopian tube. ${ }^{6}$

The probable cause of fistula in this case could be partial excision of the left horn along with the left tube and ovary, there by exposing the cavity of noncommunicating horn and leakage of menstrual blood into the peritoneal cavity. Presence of fistula can explain the symptoms of the patient, due to passage of blood into the peritoneal cavity through the fistula causing peritoneal irritation with pelvic pain and increasing dysmenorrhoea.

Transvaginal ultrasound and colour Doppler hysterosonography have been used successfully in many cases to allow direct visualization of uterine fistulas. ${ }^{3}$ Other imaging modalities include MRI with heavily T2 weighted images which show a bright fluid filled tract and CT scan. ${ }^{3}$ Case with colouterine fistula in which sonohysterography detected the flow from uterine cavity to the sigmoid colon through the posterior uterine wall, thus confirming the diagnosis. ${ }^{4}$

3D Scan and 2D power Doppler are highly accurate in detecting caesarean section scar dehiscence and uterine fistula. The diagnosis in our case was suspected on USG and MRI and confirmed by hysteroscopy and laparoscopy. The surgical approach to the management of fistulas depends on the nature of the fistulous tract, and the surgeon's experience and skill.

The successful laparoscopic repair of a uteroperitoneal fistula in a patient with endometriosis and a history of an uncomplicated caesarean delivery has been reported by M. T. Jacobson and Nezhat et al. We managed the uteroperitoneal fistula in this young girl by laparoscopic surgery with good outcome.

In experienced hands, laparoscopic management of uteroperitoneal fistuals is possible and provides the patient with the benefits of minimally invasive surgery over the traditional abdominal approach. ${ }^{7}$

\section{CONCLUSION}

Our objective is to report a rare case of uteroperitoneal fistula caused after laparoscopic left salpingo oophorectomy, during which the left non-communicating horn was opened and left a uteroperitoneal fistula.

In experienced hands, laparoscopic management of uteroperitoneal fistula is possible and laparoscopic detection and excision has provided good symptomatic relief in our patient.

Funding: No funding sources

Conflict of interest: None declared

Ethical approval: The study was approved by the Institutional Ethics Committee

\section{REFERENCES}

1. Ibinaiye PO, Onwuhafua P, Usman B. Uteroperitoneal fistula, a rare complication of laparoscopic myomectomy scar dehiscence: a case Report. The Nigerian Postgrad Medi J. 2013;20:165-6.

2. Porcaro AB, Zicari M, Antoniolli SZ, Pianon R, Monaco C, Migliorini F, et al: Vesico-uterus fistulas following caesarean section: report on a case, review and update of the literature. Int Urol Nephrol. 2002; 34:335-44.

3. Royo P, Manero MG, Olartecoechea B, Alcázar JL Two-dimensional power Doppler-three-dimensional ultrasound imaging of a caesarean section dehiscence with utero-peritoneal fistula: a case report. J Medic Case Reports. 2009,3:45.

4. Takada T, Nakagawa S, Hashimoto K, Sone K, Kugu $\mathrm{K}$, Kozuma S. et al: Preoperative diagnosis of colouterine fistula secondary to diverticulitis by sonohysterography with contrast medium. Ultrasound Obstet Gynecol. 2004;24:682-3.

5. Donnez O, Jadoul P, Squifflet J, Donnez J. Unusual complication after uterine artery embolization and laparoscopic myomectomy in a woman wishing to preserve future fertility. Fertil Steril. 2008;90:2007e5. 
6. Bhanap PL, Narwade RG: Uteroperitoneal fistula- a rare cause of failure of sterilization. Medpulse-Int Medic J. 2014;1:204-5.

7. Jacobson MT, Osias J, Velasco A, Charles R, Nezhat C. Laparoscopic repair of a uteroperitoneal fistula.
Kournal Society Laparoendoscopic Surgeons 2003,7:367-9.

Cite this article as: Swapna YK, Yelamanchi SD, Talipineni KN, Jyothsna P. A rare case of utero peritoneal fistula. Int J Reprod Contracept Obstet Gynecol 2018;7:2042-5. 